\title{
Perfil dos usuários do serviço de educação física em (1) uma Unidade Básica de Saúde
}

\author{
Profile of users of service physical education in Health Basic Unit
}

\begin{tabular}{l} 
AUTORES \\
\hline Angélica Milech $^{1}$ (D) \\
Vítor Häfele $^{1}$ (D) \\
Fernando Vinholes Siqueira $^{1}$ (D) \\
1 Universidade Federal de Pelotas, Departamento de \\
Educação Física, Pelotas, Rio Grande do Sul, Brasil. \\
CONTATO \\
Angélica Milech \\
angelicamilech@hotmail.com \\
Avenida Sargento Hermínio 2755, Apt. 301, \\
Bloco M, Fortaleza, Ceará, Brasil. \\
CEP: 60320-105. \\
DOI
\end{tabular}

$10.12820 /$ rbafs. $23 \mathrm{e} 0037$

Copyright: This is an open-access article distributed under the terms of the Creative Commons Attribution License ${ }^{\circledR}$, which permits unrestricted use, distribution, and reproduction in any medium, provided that the original author and source are credited.

\begin{abstract}
RESUMO
O objetivo do estudo foi descrever o perfil dos usuários de um serviço de educação física em uma Unidade Básica de Saúde do município de Pelotas. Os dados fazem parte do atendimento ambulatorial do profissional de educação física aos usuários de uma Unidade Básica de Saúde. Foram coletados dados sobre características demográficas, comportamentais, de saúde e de participação no atendimento ambulatorial. A amostra foi composta por 218 usuários que foram atendidos por profissional de educação física. A maioria dos usuários eram mulheres (84,4\%), com faixa etária de 50 anos ou mais $(63,4 \%)$, com hipertensão $(58,8 \%)$, com dores $(86,9 \%)$, obesos $(59,8 \%)$, insuficientemente ativos no lazer $(80,7 \%)$, que ingeria no mínimo um medicamento de maneira contínua $(80,2 \%)$, que mencionaram ter espaço próximo da residência para praticar atividade física $(62,1 \%)$ e que gostariam de realizar caminhadas $(53,6 \%)$. Conclui-se que o perfil de atendimento ambulatorial na unidade básica de saúde com profissional de educação física é composto por mulheres, por pessoas com 50 anos ou mais e por usuários com algum problema de saúde.
\end{abstract}

Palavras-chave: Educação física; Centros de saúde; Promoção da saúde.

\section{ABSTRACT}

The aim of the study was to describe the profile of the users of a physical education service in a Basic Health Unit of the city of Pelotas. The data are part of the outpatient care of the physical education professional to the users of a Basic Health Unit. Data were collected on demographic, behavioral, health and participation characteristics in outpatient care. The sample consisted of 218 users who were attended by physical education professionals. The majority of users were women (84.4\%), aged 50 years or more (63.4\%), with hypertension (58.8\%), pain (86.9\%), obese (59.8\%), insufficiently active at leisure time (80.7\%), who consumed at least one continuous medication (80.2\%), who mentioned having a place near their residence to practice physical activity (62.1\%) and who would like to walking (53.6\%). It is concluded that the profile of outpatient care in the basic health unit with a physical education professional is composed of women, people aged 50 and over, and users with any health problem.

Keywords: Physical education; Health centers; Health promotion.

\section{Introdução}

O Sistema Único de Saúde (SUS) tem realizado mudanças e avanços para a população a qual depende de atendimento. A implantação da Estratégia de Saúde da Família (ESF) na atenção básica modificou o atendimento tradicional focado nos problemas de saúde individuais para um atendimento voltado à promoção da saúde da família. Esse modelo foi fortalecido pela criação dos Núcleos de Apoio à Saúde da Família (NASF), cujo preceito é o trabalho interdisciplinar em busca de uma promoção da saúde mais eficiente, e a prática de atividade física é um componente desse processo ${ }^{1}$. A inserção do profissional de educação física (PEF) em Unidade Básica de Saúde (UBS) tem sido de grande importância para os usuários que buscam atendimento e qualidade de vida ${ }^{2}$.
A prática de atividade física é um fator fundamental para a prevenção de doenças e a promoção da saúde, devendo estar inserida no contexto da atenção primária ${ }^{3}$. Os benefícios da atividade física para saúde e qualidade de vida estão amplamente documentados, inclusive para aqueles que possuem doenças crônicas ${ }^{4}$. Destaca-se também a sua importância para o controle nos níveis de glicose e pressão arterial, e na melhoria da capacidade das pessoas para realizar as tarefas diárias e aumentando a disposição ${ }^{5}$.

Os programas de atividade física desenvolvidos e a inserção do PEF nas UBS têm auxiliado na melhoria do atendimento e da saúde dos usuários, sendo que os profissionais da área da saúde relatam ter percepção positiva sobre a atuação do $\mathrm{PEF}$ em $\mathrm{UBS}^{6}$. $\mathrm{O}$ estudo de Loch et al. ${ }^{7}$, realizado em cinco UBS de Londrina, 
Paraná, identificou que a maioria dos usuários de programas de atividade física oferecidos pelo NASF foram mulheres, pessoas com mais de 50 anos e que apresentavam alguma doença. Além disso, a pesquisa apontou que os usuários relataram o programa de atividades físicas como muito bom ou bom. Estudo de revisão sistemática sobre a inserção e atuação do PEF na atenção básica apontou que as atividades mais realizadas por esse profissional foram a caminhada, os alongamentos, o fortalecimento muscular, o equilíbrio, as atividades lúdicas, as palestras e avaliação das capacidades funcionais e qualidade de vida ${ }^{8}$.

Sendo assim, conhecer qual é o perfil em relação aos fatores demográficos, comportamentais, de saúde e de participação dos usuários de UBS que são atendidos pelo PEF é fundamental, tendo em vista que essas informações poderão auxiliar o planejamento e desenvolvimento de ações que atendam às necessidades e demandas deste púbico. Este estudo tem como objetivo descrever o perfil dos usuários em relação aos aspectos demográficos, comportamentais, de saúde e de participação no atendimento ambulatorial com o PEF em uma UBS do município de Pelotas, Rio Grande do Sul.

\section{Métodos}

O presente estudo faz parte do Projeto UBS+Ativa, o qual se caracteriza como sendo uma intervenção de atividade física que atende os moradores da área de abrangência da UBS Areal Leste na cidade de Pelotas, Rio Grande do Sul. A UBS foi selecionada intencionalmente com base nos seguintes critérios: a) estar sob gestão da Universidade Federal de Pelotas; b) atender, de segunda a sexta-feira, nos turnos da manhã e da tarde; c) dispor de espaço interno suficiente para atendimento ambulatorial e externo que possibilitasse a realização de atividades físicas coletivas supervisionadas e; d) possuir cobertura de Agente Comunitário de Saúde.

O projeto apresenta três frentes de ação: I) divulgação da função do PEF na sala de espera e aconselhamento para a prática de atividade física; II) atendimento ambulatorial à população da área de abrangência; III) aulas coletivas de atividade física com o $\mathrm{PEF}^{9}$. Para esse estudo, foram utilizados os dados do atendimento ambulatorial à população da área de abrangência, compreendendo o período de agosto de 2013 a setembro de 2016.

Os profissionais de saúde da UBS Areal Leste eram estimulados a referenciarem ao PEF da UBS seus pacientes que necessitassem do atendimento ambulato- rial deste profissional. Além disso, os adultos que foram abordados na sala de espera e aqueles que participavam das sessões de atividades físicas coletivas também poderiam ser encaminhados ao atendimento ambulatorial. Para esse estudo, fizeram parte da amostra todos os usuários com 18 anos ou mais que participaram do atendimento ambulatorial com o PEF.

Durante o atendimento ambulatorial o PEF realizava uma anamnese e fazia o aconselhamento de atividades físicas. A anamnese foi composta de questionário sobre histórico de doenças, uso de medicamentos, dores no corpo, tabagismo, questionário internacional de atividades físicas - IPAQ - domínio do lazer, e hábitos relacionados à atividade física. Quanto ao aconselhamento de atividades físicas, era informando ao paciente qual tipo de atividade realizar, o local e a existência de programas nos quais poderia se engajar, como as práticas coletivas orientadas, realização de caminhadas e sessões de exercícios programadas de acordo as exigências do grupo. Mais detalhes sobre o projeto UBS+Ativa podem ser encontrados em artigo metodológico publicado anteriormente ${ }^{9}$.

As variáveis utilizadas para descrever o perfil dos usuários do atendimento ambulatorial com o PEF foram: sexo (masculino e feminino), idade (18 - 29, 30 - 39, 40 - 49, 50 - 59 e 60 anos ou mais), índice de massa corporal - IMC, coletado através do peso e da altura mensurados (normal - até $24,9 \mathrm{~kg} / \mathrm{m}^{2}$, sobrepeso $-25,0$ até $29,9 \mathrm{~kg} / \mathrm{m}^{2}$ e obeso - 30 ou mais $\mathrm{kg} /$ $\mathrm{m}^{2}$ ), tabagismo (nunca fumou, ex-fumante e fumante), atividade física de lazer (insuficientemente ativo: não praticar atividade física de lazer ou praticar menos de 150 minutos por semana, ativo: praticar 150 minutos ou mais de atividade física de lazer por semana, verificado pelo IPAQ, versão longa, seção de lazer), autopercepção de saúde (excelente, muito boa, boa, regular, ruim e muito ruim), número de medicamentos referidos para o uso contínuo (nenhum, 1 - 2 e 3 ou mais), diagnóstico médico de hipertensão arterial (não e sim), diagnóstico médico de diabetes mellitus (não e sim), diagnóstico médico referido de outras doenças (não e sim), quais doenças existentes, dor autorreferida (não e sim), quais os locais das dores, responsável pelo encaminhamento (médico, PEF aconselhando na sala de espera, nutricionista, divulgação do projeto, agente comunitário de saúde, recepcionista, parente, vizinho e assistente social), principais motivos das consultas com o PEF, espaço próximo a residência para praticar atividade física (não e sim), qual o espaço disponível para a 
prática de atividade física e tipo de atividade física que gostaria de realizar.

Para a elaboração do banco de dados foi utilizado o programa Epidata 3.1. As análises descritivas foram realizadas no pacote estatístico Stata 13.0, incluindo medidas de tendência central (média) e dispersão (desvio padrão) para as variáveis quantitativas, e de distribuição por frequência para as variáveis qualitativas.

O projeto UBS+Ativa foi aprovado pelo Comitê de Ética em Pesquisa da Escola Superior de Educação Física da Universidade Federal de Pelotas com o número do parecer 1031599 de 21 de março de 2013.

\section{Resultados}

No período de agosto de 2013 a setembro de 2016 foram atendidos 218 usuários da UBS Areal Leste da cidade de Pelotas, Rio Grande do Sul. Destes 84,4\% eram do sexo feminino, com média de idade de 53 anos $(\mathrm{DP}=14,7$ anos), 54,9\% nunca fumaram, cerca de $80 \%$ foram considerados insuficientemente ativos no lazer (< 150 minutos de atividade física por semana no lazer), $40 \%$ consideraram sua saúde como boa, muito boa ou excelente e a maioria dos indivíduos referiram utilizar, no mínimo, um medicamento de uso contínuo (80,2\%) - Tabela 1.

A prevalência de usuários que referiram possuir diagnóstico médico de hipertensão arterial foi de $58,8 \%$, enquanto $31,2 \%$ disseram ter diagnóstico médico de diabetes mellitus. Quando questionados sobre demais doenças, $60,7 \%$ relataram a presença de no mínimo uma, sendo a mais prevalente à artrose com $19,2 \%$, seguida da depressão e hipercolesterolemia com $12 \%$ cada. A maioria $(86,9 \%)$ mencionou dor autorreferida e os locais mais relatados foram à coluna (43\%), pernas $(26,9 \%)$ e joelhos $(23,1 \%)$ - Tabela 2.

Em relação ao responsável pelo encaminhamento ao PEF, 60,4\% relataram que foi o médico, seguido do aconselhamento da sala de espera (15,9\%), nutricionista $(9,8 \%)$ e através da divulgação do projeto (7,9\%). Quanto aos principais motivos das consultas com o PEF, 30,3\% foram para receber aconselhamento para a prática de atividade física, 24,3\% devido às dores, $19,1 \%$ pela busca de emagrecimento e $14,5 \%$ devido as DNCTs. Em relação ao espaço próximo à residência para praticar atividade física, $62,1 \%$ responderam possuir algum tipo de espaço. Os espaços mais citados foram praça $(41,4 \%)$, pista de caminhada $(28,7 \%)$ e rua (14,9\%). A maioria respondeu que gostaria de realizar caminhada $(53,6 \%)$ - Tabela 3.
Tabela 1 - Distribuição das variáveis demográficas, comportamentais, índice de massa corporal, autopercepção de saúde e número de medicamentos de uso contínuo dos usuários do atendimento ambulatorial com o profissional de educação física. Pelotas, Rio Grande do Sul, 2013-2016 ( $\mathrm{n}=218)$.

\begin{tabular}{|c|c|c|}
\hline Variáveis & $\mathrm{n}$ & $\%$ \\
\hline \multicolumn{3}{|l|}{ Sexo } \\
\hline Masculino & 34 & 15,6 \\
\hline Feminino & 184 & 84,4 \\
\hline \multicolumn{3}{|l|}{ Idade (anos) } \\
\hline $18-29$ & 18 & 8,7 \\
\hline $30-39$ & 21 & 10,1 \\
\hline $40-49$ & 37 & 17,8 \\
\hline $50-59$ & 55 & 26,4 \\
\hline 60 ou mais & 77 & 37,0 \\
\hline \multicolumn{3}{|l|}{ Tabagismo } \\
\hline Nunca fumou & 107 & 54,9 \\
\hline Ex-fumante & 63 & 32,3 \\
\hline Fumante & 25 & 12,8 \\
\hline \multicolumn{3}{|l|}{ Atividade física (lazer) } \\
\hline Insuficientemente ativo & 159 & 80,7 \\
\hline Ativo & 38 & 19,3 \\
\hline \multicolumn{3}{|l|}{ Índice de massa corporal } \\
\hline Normal & 18 & 16,1 \\
\hline Sobrepeso & 27 & 24,1 \\
\hline Obesidade & 67 & 59,8 \\
\hline \multicolumn{3}{|l|}{ Autopercepção de saúde } \\
\hline Excelente & 4 & 1,9 \\
\hline Muito boa & 9 & 4,3 \\
\hline Boa & 71 & 33,8 \\
\hline Regular & 102 & 48,6 \\
\hline Ruim & 18 & 8,6 \\
\hline Muito ruim & 6 & 2,9 \\
\hline \multicolumn{3}{|c|}{ Número de medicamentos referidos para uso contínuo } \\
\hline Nenhum & 40 & 19,8 \\
\hline $1-2$ & 75 & 37,1 \\
\hline 3 ou mais & 87 & 43,1 \\
\hline
\end{tabular}

\section{Discussão}

Os principais achados do estudo demonstram que o atendimento ambulatorial com o PEF tem maior participação das mulheres, pessoas com 50 anos ou mais, com algum problema de saúde, que utilizam algum medicamento, considerados insuficientemente ativos no lazer, que mencionam ter espaço próximo da residência para praticar atividade física e que gostariam de realizar caminhadas.

Os resultados do estudo mostram que, a maioria dos usuários foram mulheres e a média de idade foi de 53 anos. Há vários estudos com usuários de UBS que mostram a similaridade entre as características sexo e idade ${ }^{10,11}$. A carência dos homens no atendimento 
Tabela 2 - Prevalência de morbidades e dor autorrelatadas dos usuários do atendimento ambulatorial com o profissional de educação física. Pelotas, Rio Grande do Sul, 2013-2016 ( $\mathrm{n}=218$ ).

\begin{tabular}{|c|c|c|}
\hline Variáveis & $\mathrm{n}$ & $\%$ \\
\hline \multicolumn{3}{|l|}{ Hipertensão arterial* } \\
\hline Não & 87 & 41,2 \\
\hline Sim & 124 & 58,8 \\
\hline \multicolumn{3}{|l|}{ Diabetes mellitus* } \\
\hline Não & 143 & 68,8 \\
\hline Sim & 65 & 31,2 \\
\hline \multicolumn{3}{|c|}{ Outras doenças referidas* } \\
\hline Não & 81 & 39,3 \\
\hline $\operatorname{Sim}$ & 125 & 60,7 \\
\hline \multicolumn{3}{|l|}{ Quais doenças ${ }^{\#}$} \\
\hline Artrose & 24 & 19,2 \\
\hline Depressão & 15 & 12,0 \\
\hline Hipercolesterolemia & 15 & 12,0 \\
\hline Osteoporose & 14 & 11,2 \\
\hline Hipotireoidismo & 12 & 9,6 \\
\hline \multicolumn{3}{|l|}{ Dor autorreferida } \\
\hline Não & 28 & 13,1 \\
\hline Sim & 186 & 86,9 \\
\hline \multicolumn{3}{|l|}{ Locais das dores } \\
\hline Coluna & 80 & 43,0 \\
\hline Perna & 50 & 26,9 \\
\hline Joelho & 43 & 23,1 \\
\hline Ombro & 22 & 11,8 \\
\hline Pé & 19 & 10,2 \\
\hline
\end{tabular}

* Diagnóstico médico referido. \# Cinco doenças mais prevalentes dos indivíduos que apresentaram outras doenças. \#\# Cinco locais de dores mais prevalentes dos indivíduos que apresentaram dores autorrelatadas.

ambulatorial pode estar relacionada à mulher se preocupar mais com a saúde e estar vinculada desde cedo na UBS com consultas periódicas ao ginecologista e posteriormente, por questões obstétricas ${ }^{12}$. Além disso, sabe-se que os homens evitam a procura da atenção básica, buscando a atenção especializada quando percebem que possuem algum tipo de problema de saúde. Com isso, foi criada no ano de 2008 a Política Nacional de Atenção Integral à Saúde do Homem, visando uma assistência integral a essa população, com ênfase nas questões de prevenção, promoção e proteção à saúde ${ }^{13}$.

Em relação ao IMC, a maior parte dos usuários foram considerados obesos, assim como no estudo de Turi et al. ${ }^{14}$. Devido aos riscos da obesidade, os quais podem provocar o aumento de DCNTs, artrose e aumento do $\mathrm{IMC}^{15}$, é importante a presença do PEF nas UBS para auxiliar na promoção de estilos de vida mais saudáveis para esta população.

Cerca de $80 \%$ dos usuários relataram ser insufi-
Tabela 3 - Características da participação no atendimento ambulatorial com o profissional de educação física. Pelotas, Rio Grande do Sul, 2013-2016 ( $\mathrm{n}=218)$.

\begin{tabular}{|c|c|c|}
\hline Variáveis & $\mathrm{n}$ & $\%$ \\
\hline \multicolumn{3}{|l|}{ Responsável pelo encaminhamento } \\
\hline Médico & 99 & 60,4 \\
\hline PEF aconselhando sala de espera & 26 & 15,9 \\
\hline Nutricionista & 16 & 9,8 \\
\hline Divulgação do projeto & 13 & 7,9 \\
\hline Agente comunitário de saúde & 3 & 1,8 \\
\hline Recepcionista & 3 & 1,8 \\
\hline Parente & 2 & 1,2 \\
\hline Vizinho & 1 & 0,6 \\
\hline Assistente social & 1 & 0,6 \\
\hline \multicolumn{3}{|l|}{$\begin{array}{l}\text { Principais motivos das consultas com o profissional de } \\
\text { educação física\# }\end{array}$} \\
\hline $\begin{array}{l}\text { Receber aconselhamento para a prática de atividade } \\
\text { física }\end{array}$ & 46 & 30,3 \\
\hline Dores & 37 & 24,3 \\
\hline Emagrecer & 29 & 19,1 \\
\hline Doenças crônicas não transmissíveis & 22 & 14,5 \\
\hline Devido ao encaminhamento & 11 & 7,2 \\
\hline \multicolumn{3}{|l|}{ Espaço próximo à residência para praticar atividade física } \\
\hline Não & 53 & 37,9 \\
\hline Sim & 87 & 62,1 \\
\hline \multicolumn{3}{|l|}{ Qual espaço } \\
\hline Praça & 36 & 41,4 \\
\hline Pista de caminhada & 25 & 28,7 \\
\hline Rua & 13 & 14,9 \\
\hline Campo de futebol & 9 & 10,3 \\
\hline Pátio & 4 & 4,6 \\
\hline Estação $1^{\circ}$ do Areal & 3 & 3,4 \\
\hline Academia & 2 & 2,3 \\
\hline Núcleo da Brigada Militar & 2 & 2,3 \\
\hline \multicolumn{3}{|l|}{ Tipo de atividade física que gostaria de realizar } \\
\hline Caminhada & 81 & 53,6 \\
\hline Ciclismo & 31 & 20,5 \\
\hline Ginástica & 18 & 11,9 \\
\hline Futebol & 10 & 6,6 \\
\hline Dança & 11 & 7,3 \\
\hline Academia & 11 & 7,3 \\
\hline Qualquer atividade física & 7 & 4,6 \\
\hline Não sabe & 9 & 6,0 \\
\hline
\end{tabular}

\# Cinco motivos mais prevalentes das consultas com o profissional de educação física.

cientemente ativos no lazer. O estudo de Siqueira et al. ${ }^{16}$, realizado nas regiões Sul e Nordeste do Brasil, demonstrou que $31,8 \%$ dos adultos e $58 \%$ dos idosos foram considerados insuficientemente ativos no tempo de lazer. Essa diferença nos resultados pode ser atribuída ao fato de que no estudo de Siqueira et al. foi utilizada a versão curta do IPAQ, enquanto no presen- 
te estudo foi aplicada a seção de lazer da versão longa do IPAQ. Neste sentido, com os dados demonstrando baixos níveis de atividade física em usuários de UBS, é necessário desenvolver e ampliar ações de promoção da atividade física na atenção básica, o que reforça a importância do PEF na UBS.

Foi verificado que cerca de $13 \%$ dos sujeitos eram fumantes, sendo o resultado inferior ao encontrado por Siqueira et al. ${ }^{16}$, o qual apontou que $27,7 \%$ dos adultos e $15,2 \%$ dos idosos usuários de UBS eram fumantes. $\mathrm{O}$ uso de tabaco está diminuindo com o passar dos anos ${ }^{17}$ devido a diversas ações desenvolvidas, como a proibição de fumar em locais fechados, o uso de cartazes de proibição, o aumento do preço do produto, advertências nos rótulos e proibições de propagandas que induzem ao consumo ${ }^{18 .}$ No presente estudo, o fato de grande parte dos usuários nunca ter fumado ou terem parado de fumar deve ser considerado positivo, tendo em vista que o tabagismo é um dos fatores de risco para diversas doenças, como doenças respiratórias, cardiovasculares, além de trazer problemas bucais e ser um fator de risco para o aumento da morbimortalidade ${ }^{19,20}$.

Em relação às doenças autorrelatadas dos usuários do atendimento ambulatorial com o PEF, observou-se elevada prevalência de hipertensão arterial $(58,8 \%)$ e diabetes mellitus $(31,2 \%)$. Esses resultados foram superiores aos encontrados em pesquisa realizada com usuários em geral de 240 UBS das regiões Sul e Nordeste do Brasil ${ }^{21}$, fato esse que demonstra que os usuários muitas vezes procuram o PEF na UBS para tratamento de problemas de saúde ao invés da prevenção.

Além da hipertensão e da diabetes, 60,7\% dos usuários do atendimento ambulatorial mencionaram possuir alguma outra doença, das quais as mais citadas foram: artrose (19,2\%), depressão (12\%), hipercolesterolemia (12\%), osteoporose $(11,2 \%)$ e hipotireoidismo (9,6\%). Esses achados são similares aos do estudo de Malta et al. ${ }^{22}$, que verificaram que as doenças mentais, a artrite e as dores de coluna foram os problemas mais prevalentes na população brasileira. As DCNTs estão entre as maiores causas de mortalidade no Brasil afetando grande parte da população ${ }^{23}$. Nesse sentido, tornam-se importantes ações de proteção e promoção da saúde nas $\mathrm{UBS}^{24}$, com as equipes multiprofissionais (incluindo o PEF) planejando e executando atividades que possam contribuir para uma mudança do estilo de vida da população.

Dos usuários, $86,9 \%$ referiram algum tipo de dor, sendo a grande parte das dores localizadas na coluna
(43\%). Ferreira et al. ${ }^{25}$ verificaram que $63,1 \%$ dos adultos de um município de médio porte do Sul do Brasil possuíam dor nas costas. As dores na região lombar estão cada vez mais frequentes e fatores como a idade avançada e o convívio com o problema acabam agravando a dor ${ }^{26}$. Nesse contexto, a prática de atividade física pode ser utilizada como um tratamento terapêutico para o alívio das dores e também como um meio de prevenção para o surgimento de tal problema ${ }^{27}$.

Em relação as características da participação no atendimento ambulatorial com o PEF, o médico foi o principal agente no encaminhamento para a consulta com o PEF, seguido das atividades de aconselhamento na sala de espera da UBS, nutricionista, divulgação do projeto UBS+Ativa e agente comunitário de saúde, mostrando a importância de vários profissionais de diferentes áreas da saúde trabalhando em conjunto buscando um estilo de vida saudável para os usuários de modo multiprofissional ${ }^{28}$.

Os principais motivos relatados pelos usuários para a consulta com o PEF foram à busca de aconselhamento para a prática de atividade física, seguido de dores, emagrecimento, DCNT e devido ao encaminhamento. Esses resultados reforçam a importância da inserção do PEF nas UBS tendo em vista que o mesmo pode auxiliar na melhoria da qualidade de vida da população. Além disso, a busca de atendimento por parte dos usuários com o PEF mostra a preocupação com a saúde e reconhecimento da importância da prática de atividade física.

A maioria dos usuários mencionou ter espaço próximo à residência para a prática de atividade física, sendo os locais mais citados: praça, pista de caminhada e rua. Esse achado é relevante no sentido de que os usuários do SUS estão percebendo que locais públicos são possíveis de ser utilizados para a prática de atividade física. O PEF deve incentivar e orientar os usuários para que utilizem tais espaços públicos para a prática de atividade física. Sendo assim, cabe ao poder público investir na manutenção e na melhoria desses espaços, como paisagismo, iluminação, calçadas adequadas, ciclovias, parques urbanos, sinalização e segurança.

A caminhada foi a atividade física mais citada pelos usuários em relação a atividade que mais gostaria de realizar. A caminhada é a atividade mais praticada no Brasil, tanto pelas mulheres quanto pelos homens e com o aumento da idade o percentual eleva ${ }^{29}$. Diversos são os benefícios provenientes dessa prática, como a prevenção ou tratamento de DCNT, melhoria da saúde mental e interação social ${ }^{30}$. Outro fator relevante em relação a ca- 
minhada refere-se a acessibilidade, tendo em vista que é uma atividade apropriada independentemente da idade ou renda, sem a necessidade de equipamentos, habilidades específicas ou local próprio, favorecendo a redução de desigualdades e de falta de atividade física ${ }^{30}$.

As principais limitações do estudo foram: a pesquisa realizada em apenas uma UBS e com uma amostra não representativa, não permitindo extrapolar os achados para outras UBS. Como ponto forte do estudo encontram-se as entrevistas que foram realizadas em formato de anamnese por um PEF.

Conclui-se que a maioria dos pacientes atendidos pelo PEF foram mulheres, com faixa etária de 50 anos ou mais, com hipertensão, com dores, obesos, insuficientemente ativos no lazer, que ingerem no mínimo um medicamento de maneira contínua, que mencionam ter espaço próximo da residência para praticar atividade física e que gostariam de realizar caminhadas. Os resultados obtidos servem como base para os PEF que estão inseridos em UBS, além de servir para qualificar as equipes multiprofissionais que atuam em UBS, visando o desenvolvimento do tema atividade física na atenção primária à saúde.

\section{Conflito de interesse}

Os autores declaram não haver conflito de interesse.

\section{Contribuição dos autores}

Milech A, participou de todas as fases do artigo. Häfele V, auxiliou na redação do artigo, na análise dos dados e na revisão final do artigo. Siqueira FV, participou na qualidade de revisor final de todas as etapas, desde a elaboração do projeto até a redação final do artigo. Todos os autores contribuíram para a escrita do artigo.

\section{Financiamento}

Agradecemos ao apoio financeiro concedido pela FAPERGS.

\section{Referências}

1. Florindo AA. Núcleos de Apoio a Saúde da Família e a promoção das atividades físicas no Brasil: De onde viemos, onde estamos e para onde vamos. Rev Bras Ativ Fís Saúde. 2009;14(1):72-3.

2. Lucena D, Borges KEL, Aguiar MA, Alcântara F, Madruga JG, Lomeo R. A inserção da educação física na estratégia saúde da família em Sobral/CE. Rev Polit Públ-Sanare. 2004;5(1):87-1.

3. Siqueira FV, Nahas MV, Facchini LA, Silveira DS, Piccini RX, Tomasi E, et al. Aconselhamento para a prática de atividade física como estratégia de educação à saúde. Cad Saude Publica. 2009;25(1):203-13.

4. Warburton DE, Nicol CW, Bredin SS. Health benefits of physical activity: the evidence. CMAJ. 2006;174(6):801-09.
5. Kokubun E, Luciano E, Sibuya CY, Queiroga MR, Ribeiro $\mathrm{PAB}$, Silveira RF, et al. Programa de atividade física em unidades básicas de saúde: Relato de experiência no município de Rio Claro-SP. Rev Bras Ativ Fís Saúde. 2007;12(1):45-53.

6. Pedrosa OP, Leal AF. A inserção do profissional de educação física na estratégia saúde da família em uma capital do norte do Brasil. Rev Movimento. 2012;18(2):235-53.

7. Loch MR, Rodrigues CG, Teixeira DC. E os homens? E os que moram longe? E os mais jovens?...? Perfil dos usuários de programas de atividade física oferecidos pelas unidades básicas de saúde de Londrina-PR. Rev Bras Ciênc Esporte. 2013;35(4):947-61.

8. Rodrigues JD, Ferreira DKS, Silva PA, Caminha IO, Farias Júnior JC. Inserção e atuação do profissional de educação física na atenção básica à saúde: revisão sistemática. Rev Bras Ativ Fís Saúde. 2013;18(1):5-15.

9. Galliano L, Seus T, Peixoto M, Silva W, Silveira D, Del Vecchio F, et al. Intervenção com atividade física em uma Unidade Básica de Saúde - Projeto UBS+Ativa: Aspectos metodológicos. Rev Bras Ativ Fís Saúde. 2016;21(6):571-80.

10. Fernandes LCL, Bertoldi AD, Barros AJD. Utilização dos serviços de saúde pela população coberta pela Estratégia de Saúde da Família. Rev Saude Publica. 2009;43(4):595-03.

11. Pimentel IRS, Coelho BC, Lima JC, Ribeiro FG, Sampaio FPC, Pinheiro RP, et al. Caracterização da demanda em uma Unidade de Saúde da Família. Rev Bras Med Fam Comunidade. 2011;6(20):175-81.

12. Costa-Júnior FM, Maia ACB. Concepções de Homens Hospitalizados sobre a Relação entre Gênero e Saúde. Psic: Teor e Pesq. 2009;25(1):55-63.

13. Brasil. Ministério da Saúde. Secretaria de Atenção à Saúde. Política Nacional de Atenção Integral à Saúde do Homem: princípios e diretrizes. Brasília, p. 92, 2009.

14. Turi BC, Codogno JS, Fernandes RA, Monteiro HL. Prática de atividade física, adiposidade corporal e hipertensão em usuários do Sistema Único de Saúde. Rev Bras Epidemiol. 2014;17(4):925-37.

15. World Health Organization. Global status report on noncommunicable disease 2010. Geneva: WHO; 2011.

16. Siqueira FV, Facchini LA, Piccini RX, Tomasi E, Thumé E, Silveira DS, et al. Atividade física em adultos e idosos residentes em áreas de abrangência de unidades básicas de saúde de municípios das regiões Sul e Nordeste do Brasil. Cad Saude Publica. 2008;24(1):39-54.

17. Malta DC, Stopa SR, Iser BPM, Bernal RTI, Claro RM, Nardi ACF, et al. Fatores de risco e proteção para doenças crônicas por inquérito telefônico nas capitais brasileiras, Vigitel 2014. Rev Bras Epidemiol. 2015;18(2):238-55.

18. Brasil. Presidência da República/Casa Civil Decreto no 8.262, de 31 de maio de 2014.

19. Rodrigues ESR, Cheik NC, Mayer AF. Nível de atividade física e tabagismo em universitários. Rev Saude Publica. 2008;42(4):672-8.

20. Moreira LB, Fuchs FD, Moraes RS, Bredemeir M, Cardozo S. Prevalência de tabagismo e fatores associados em área metropolitana da região Sul do Brasil. Rev Saude Publica. 1995;29(1):46-51.

21. Facchini LA, Piccini RX, Tomasi E, Thumé E, Silveira DS, Siqueira FV, Rodrigues MA. Desempenho do PSF no Sul e no Nordeste do Brasil: avaliação institucional e epidemiológica da Atenção Básica à Saúde. Ciênc Saúde Colet. 2006;11(3):669-81. 
22. Malta DC, Stopa SR, Szwarcwald CL, Gomes NL, Silva Júnior JB, Reis AAC. A vigilância e o monitoramento das principais doenças crônicas não transmissíveis no BrasilPesquisa Nacional de Saúde, 2013. Rev Bras Epidemiol. 2015;18(2):3-16.

23. Schmidt MI, Duncan BB, Hoffmann JF, Moura L, Malta DC, Carvalho RMSV. Prevalência de diabetes e hipertensão no Brasil baseado em inquérito de morbidade auto-referida, Brasil, 2006. Rev Saude Publ. 2009;43(2):74-82.

24. Malta DC, Cezário AC, Moura L, Morais Neto OL, Silva Júnior JB. A construção da vigilância e prevenção das doenças crônicas não transmissíveis no contexto do Sistema Único de Saúde. Epidemiol Serv Saúde. 2006;15(3):47-65.

25. Ferreira GD, Silva MC, Rombaldi AJ, Wrege ED, Siqueira FV, Hallal PC. Prevalência de dor nas costas e fatores associados em adultos do Sul do Brasil: estudo de base populacional. Rev Bras Fisioter. 2011;15(1):31-6.

26. Silveira AIP, Oliveira JA, Silva PTC, Lopes RA, Coelho MM. Correlação entre relato de dor lombar crônica, fatores psicossociais e capacidade física em idosos comunitários. Fisioter Bras. 2014;15(4):277-82.
27. Holth HS, Werpen HKB, Zwart J-A, Hagen K. Physical inactivity is associated with chronic musculoskeletal complaints 11 years later: results from the Nord-Trøndelag Health Study. BMC Musculoskelet Disord. 2008;9(159):1-7.

28. Barbosa VLP, Cézar C, Vítolo MR, Lopez FA. Atuação ambulatorial do profissional de educação física no atendimento a crianças e adolescentes obesos. Rev Bras Med Esporte. 1999;5(1):31-34.

29. Malta DC, Moura EC, Castro AM, Cruz DKA, Morais Neto OL, Monteiro CA. Padrão de atividade física em adultos brasileiros: resultados de um inquérito por entrevistas telefônicas, 2006. Epidemiol Serv Saúde. 2009;18(1):7-16.

30. Lee IM, Buchner DM. The importance of walking to public health. Med Sci Sports Exerc. 2008;40(7 Suppl):S512-8.

Recebido: 03/08/2018

Aprovado: 01/11/2018

\section{Como citar este artigo:}

Milech A, Häfele V, Siqueira FV, Perfil dos usuários do serviço de educação física em uma Unidade Básica de Saúde. Rev Bras Ativ Fís Saúde. 2018;23:e0037. DOI: 10.12820/rbafs.23e0037 